\title{
3D models related to the publication: Internal tooth structure and burial practices: insights into the Neolithic necropolis of Gurgy (France, 5100-4000 cal. BC).
}

\author{
Le Luyer Mona $^{1 *}$, Coquerelle Michael ${ }^{2}$, Rottier Stéphane ${ }^{1}$, Bayle Priscilla ${ }^{1}$ \\ 1 UMR 5199 PACEA, Université de Bordeaux, CNRS, MCC, Bâtiment B8, Allée Geoffroy Saint Hilaire, CS 50023, 33615 Pessac Cedex, France \\ ${ }^{2}$ Department of Oral Surgery, Rey Juan Carlos University, Alcorcon, Spain \\ *Corresponding author: mona.le-luyer@u-bordeaux.fr
}

\begin{abstract}
The present 3D Dataset contains the 3D models of external and internal aspects of human upper permanent second molars from the Neolithic necropolis analyzed in the following publication: Le Luyer M., Coquerelle M., Rottier S., Bayle P., 2016. Internal tooth structure and burial practices: insights into the Neolithic necropolis of Gurgy (France, 5100-4000 cal. BC). Plos One 11(7): e0159688. doi: 10.1371/journal.pone.0159688
\end{abstract}

Keywords: modern humans, Neolithic, upper permanent second molars

Submitted:2016-03-13, published online:2016-25-07. doi: 10.18563/m3.2.1.e1

\section{INTRODUCTION}

The 3D models correspond to virtually reconstructed crowns of upper permanent second molars (UM2) from 20 Neolithic humans of the necropolis of Gurgy (France) (see Table 1). Crown tissue proportions, thickness and distribution of enamel, as well as enamel-dentine junction shape were assessed to characterize subtle phenotypic dental variation and its underlying causes among individuals buried at Gurgy. In order to finely quantify size and shape variations in a microevolutionary context, original methods and templates were developed. The results show that intrasite dental variation reflects burial practices and chronocultural parameters. Underlying causes of these internal tooth structure variations were suggested (see Le Luyer et al., 2016).

\section{METHODS}

Following the half-maximum height method (Coleman \& Colbert, 2007; Spoor et al., 1993), a semi-automatic thresholdbased segmentation with manual corrections was conducted using Avizo 7.0 (Visualization Sciences Group). The 3D surface models were generated using a constrained smoothing algorithm. For each specimen, the outer enamel surface (OES) and the enamel-dentine junction (EDJ) are provided in .ply format, and can therefore be opened with a wide range of freeware. For slightly worn teeth, reconstructions of the apex of dentine horn tips were made using Avizo 7.0 (Visualization Sciences Group).

\section{ACKNOWLEDGEMENTS}

This study was supported by the Research National Agency through the DHP project (dir: S. Rottier; 2012-14; Université Bordeaux 1/LaScArBx; Grant number: ANR-10-LABX-52) and the PEPS 3Dent' in (dir: P. Bayle; 2013-14; PEPS IdEx Bordeaux/CNRS; Grant number: ANR-10-IDEX-03-02). M. Le Luyer benefited from a doctoral grant of the Ministère de l'Enseignement Supérieur et de la Recherche. MicroCT data presented in this work were produced through the technical facilities of the MRI platform and of the labEx CeMEB. We are grateful to Renaud Lebrun.

\section{BIBLIOGRAPHY}

Coleman, M. N., Colbert, M. W., 2007. Technical note: CT thresholding protocols for taking measurements on threedimensional models. Am J Phys Anthropol 133(1), 723-725. doi: 10.1002/ajpa.20583.

Spoor, F., Zonneveld, F., Macho, G. A., 1993. Linear measurements of cortical bone and dental enamel by computed tomography: applications and problems. Am J Phys Anthropol 91(4), 469-484. doi: 10.1002/ajpa.1330910405.

Le Luyer M., Coquerelle M., Rottier S., Bayle P., 2016. Internal tooth structure and burial practices: insights into the Neolithic necropolis of Gurgy (France, 5100-4000 cal. BC). Plos One 11(7): e0159688. doi: 10.1371/journal.pone.0159688. 


\section{Model IDs \\ M3\#75_GLN04-206-ULM2 \\ M3\#74_GLN04-201-ULM2 \\ M3\#76_GLN05-213-URM2 \\ M3\#77_GLN05-215A-URM2 \\ M3\#78_GLN06-215B-URM2 \\ M3\#79_GLN06-223-URM2 \\ M3\#80_GLN04-229-URM2 \\ M3\#81_GLN05-243B-ULM2 \\ M3\#82_GLN04-248-ULM2 \\ M3\#83_GLN04-252-ULM2 \\ M3\#84_GLN04-253-ULM2 \\ M3\#85_GLN05-257-URM2 \\ M3\#86_GLN04-264-ULM2 \\ M3\#87_GLN04-277-URM2 \\ M3\#88_GLN04-289B-URM2 \\ M3\#89_GLN06-291-URM2 \\ M3\#90_GLN05-292-URM2 \\ M3\#91_GLN05-294-ULM2 \\ M3\#92_GLN05-301-ULM2 \\ M3\#93_GLN05-308-URM2}

Taxon

Homo sapiens

Homo sapiens

Homo sapiens

Homo sapiens

Homo sapiens

Homo sapiens

Homo sapiens

Homo sapiens

Homo sapiens

Homo sapiens

Homo sapiens

Homo sapiens

Homo sapiens

Homo sapiens

Homo sapiens

Homo sapiens

Homo sapiens

Homo sapiens

Homo sapiens

Homo sapiens

\section{Short description}

OES and EDJ of left UM2

OES and EDJ of left UM2

OES and EDJ of right UM2

OES and EDJ of right UM2

OES and EDJ of right UM2

OES and EDJ of right UM2

OES and EDJ of right UM2

OES, EDJ and reconstructed dentine horn tip of left UM2

OES, EDJ and reconstructed dentine horn tip of left UM2

OES and EDJ of left UM2

OES and EDJ of left UM2

OES, EDJ and reconstructed dentine horn tip of right UM2

OES and EDJ of left UM2

OES and EDJ of right UM2

OES and EDJ of right UM2

OES, EDJ and reconstructed dentine horn tip of right UM2

OES and EDJ of right UM2

OES, EDJ and reconstructed dentine horn tip of left UM2

OES and EDJ of left UM2

OES and EDJ of right UM2

Table 1. List of models. OES: outer enamel surface. EDJ: enamel-dentine junction. UM2: upper permanent second molar 